\title{
Research on Key Technologies of communication and computing in Smart Grid
}

\author{
OU Qinghai ${ }^{\mathrm{a}, 1}$, HE Qingsu ${ }^{\mathrm{b}, 1}$, XU shiliang ${ }^{\mathrm{c}, 1}$, ZENG Lingkang ${ }^{\mathrm{d}, 1}$, LIAO Xiao ${ }^{\mathrm{e}, 1}$ \\ ${ }^{1}$ State Grid Information \& Telecommunication Group. CO. LTD, Beijing 100031, China; \\ ${ }^{2}$ Beijing Huitong Financial Information Technology CO.LTD,Beijing 100053,China; \\ ${ }^{3}$ Beijing University of Chemical Techonology, Beijing, 100029, China \\ ae-mail: ouqinghai@sgitg.sgcc.com.cn, be-mail: heqingsu@sgitg.sgcc.com.cn
}

Keywords: Smart Grid; Communication scheduling; Collaborative computing; simulation platform

\begin{abstract}
Research on the data model of intelligent power distribution system and study the typical characteristics. Proposed the communication scheduling model of smart distribution network communication system, and find the node model to enhance the capabilities of data synchronization processing in the case of a sharp rise in the amount of data nodes of data. Then, research on the key technology and explore the theory of the collaboration of communicate scheduling and computing power. To build co-operation models of the entire system of intelligent distribution communications and computing. On this basis, going further explore distributed intelligent data processing technology and device virtualization. In the end, to establish a collaborative simulation platform of communication and calculation in the typical application of the intelligent distribution network environment. To test the results, meanwhile we should provide simulation experiment platform to against the adjustments and changes would be happen in the development of intelligent distribution network.
\end{abstract}

\section{Introduction}

Along with the construction and development of the power system primary equipment, the second system construction and the communication automation technology, the control SCADA system of the power grid is becoming more and more mature. To realize automation control and deep effect on the production and operation of power grid construction, and greater economic benefits, to actively promote everything in good order and well arranged "strong smart grid" construction, will inevitably face some problems become increasingly prominent in the new development conditions. For intelligent distribution network communication network, intelligent distribution network system platform and based on the development and upgrade of distribution network communication system and business, these problems can't be ignored.

First, the intelligent distribution network communication system takes on a lot of business data transmission in the terminals of the intelligent network [1]. With the wide access of new energy power, the distribution network is connected with renewable power, and the data for the dispatch, control and management of micro network system is necessary for the distribution network communication system. At the same time, carrying out the user side demand management and the use of electric information monitoring, also need the data based on the user and the system of two-way interactive .The data also increased the load of distribution network communication system. Another important demand reflected in the traditional network equipment maintenance cycle model to condition based maintenance mode transition process, inevitably need to support the equipment monitoring and control functions of sensing data were collection, transmission, bearing mass sensing data transmission with network communication system will be faced with large load and high concurrency problem [2].

The actual development needs contribute to the requirements for the difference of the distribution network communication system in wide area communication network and grid SCADA communication system (relatively smaller amount of data load and high reliability) special functions and features. The intelligent distribution network communication system faces several 
problems which need to be solved urgently:

1) To establish a communication computing collaboration model and network performance evaluation method

2) Heterogeneous network fusion

3) Distributed collaborative computing model

4) Virtualization technology for communication and computing resources

The paper based on the basic theory and key technology research, combined with the actual situation of communication, data statistics and business data characteristics, put forward the corresponding solutions.

\section{Typical business data of intelligent distribution network}

Smart grid is based on the physical network, makes full use of modernization of sensor technology, communication technology, computer technology, information technology, control technology and new energy technology, the generation, transmission, distribution, link connectivity, becomes the height of the new intelligent network [3]. Smart grid as a self-healing grid, power grid to predict possible problems, can carry out continuous on-line self-assessment, implementation of existing or evolving problems quickly found, and immediately take measures to control and correct. In order to guarantee the reliability, security, power quality and efficiency of power network. Smart grid will withstand the attack, to maximize the reduction of power grid disturbance, to reduce the impact on economic development.

According to the current situation of the power grid, the typical business data of smart distribution network mainly includes:

1) Distributed energy into the distribution network, its scheduling, control, monitoring, management, resulting in a large number of data.

"Distributed energy" is a comprehensive utilization system of energy distributed on the user side. Fuel gas is the primary energy, renewable energy as a supplement, using all available resources [4]; it also includes the various energy storage energy equipment, such as a battery storage. The communication demand of the distributed energy station system mainly includes the dispatching, controlling, monitoring and management of the energy station, and the communication bandwidth of each energy station is about $64 \mathrm{k}-1 \mathrm{Mkps}$.

2) A large amount of data generated by the electric quantity collection service.

Electricity acquisition mainly includes large customers' electricity consumption, ordinary residents' electricity consumption, mark for the electricity, in addition to the extension of the useful electrical information. The large customer is a large load of users, the special needs of users, power consumption and a greater impact on the power grid, in addition to the conventional power acquisition, still need to be load demand management. The main information includes load forecasting, power quality monitoring, conform to the control parameters and so on. Communication bandwidth is about $5 \mathrm{~K}$. Time delay is divided into level. Mark power form its real-load acquisition proceeds. Installed its real-load refers to run on inter-district tie line, provincial power companies on the Internet, network link, and let down the nets in the province level of watt-hour meter, electric energy metering device used for trade settlement and the assessment of internal economic indicators, esteem to role in the whole grid power calculation. Electricity information is on the basis of the electric power data, combining information such as time, preliminary analysis results, such as user electricity usage, etc [5].

3) Distribution network equipment and circuit of on-line monitoring, diagnosis and control of data.

For example traditional distribution automation, mainly meet of feeder terminal unit (FTU), distribution terminal unit (DTU) and transformer terminal unit(TTU) several distribution network equipment and communication between master station and automatic control of data [6].

Distribution automation data mainly came from the distribution monitoring terminal, according to GB/T 18657.5 (IEC60870-5) and maximum number of bytes per frame 256 calculation, distribution automation in the fastest 3 seconds of information change refresh requirements. The 
terminal speed is calculated as shown in the following formula.

$$
C=\left[\left(L_{1}+L_{2}\right) \times m+\left(L_{1}^{\prime}+L_{2}^{\prime}\right) \times n\right] \times B \div T
$$

Formula used in several parameters include: control application layer byte number L1, additional protocol byte number L2 , control frames m, monitoring application layer byte number L'1, additional protocol byte number L'2, monitoring frames $\mathrm{n}$, each byte bit number $\mathrm{B}$, and data transmission period $\mathrm{T}$.

Intelligent distribution network service data transmission, between the terminal and the master station is generally between the 101 protocol, between the terminal and the terminal or between the interrupt and the sub stations generally use the 101, 104 protocol, the graph model support SVG, G language, CIM, etc.

Distribution automation terminal the A+ class regional power distribution terminal type to three remote mainly, A, B and C power supply area mainly to two remote, class D power supply area mainly to two remote, remote, class E power supply area to a remote mainly [7].

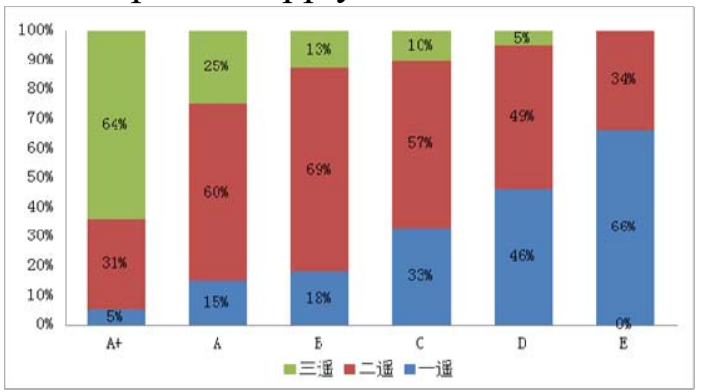

Fig. 1 Distribution terminal distribution proportion of pilot project

The construction mode of distribution network automation system mainly depends on the overall scale of the distribution network, the complexity of the wiring, the functional requirements of the automation system, the expected level of automation and so on. The communication mode adopted by different areas will have an important influence on the performance of distribution automation. Through the experiment research shows, Pole top transformers of Beijing are using wireless public network. Sub-section post adopts special way of optical fiber communication mode. Shanghai mainly adopts medium voltage power line carrier communication mode. Hangzhou, Xiamen, Yinchuan, Nanjing, Chengdu and other cities used in optical fiber communication, wireless and carrier of communication network.

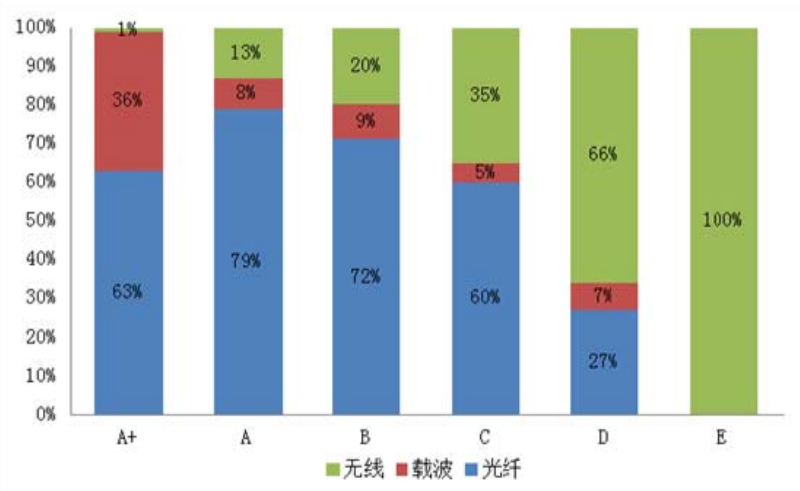

Fig. 2 Pilot project area distribution automation communication mode scale

\section{Computation and communication collaboration model for intelligent distribution network}

To meet the demand of communication and computation efficiency of collaborative goal, project specific practice process to follow the technical route as follows: Extract the existing distribution network communication system in the process of running in the state, the efficiency of the business data is loaded by the statistics and analysis of its characteristics, provided a model of coordination communication ability. The model includes five parts: business data characteristics and quantitative analysis; communication network performance evaluation; integration of heterogeneous networks; distributed collaborative computing; resource virtualization. Finally, according to the model to establish communication with computing key technologies of the simulation platform, and carry out 
the simulation of the proposed model and method. According to the gap between the simulation results and the initial data to adjust the model, and the model is gradually improved and stabilized through the mechanism of this kind of negative feedback. As shown in figure 3:

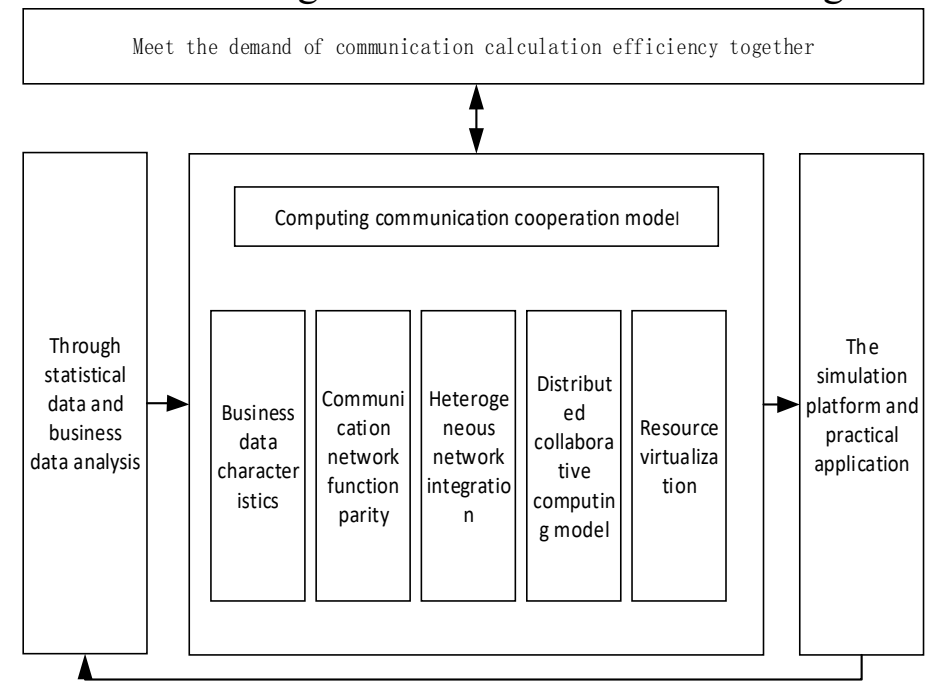

Fig. 3 Computational communication capability collaboration model

In the early application of automation system, the computing power is not a prominent and did not need to pay attention. The reason is that early system capacity and communication scales for computational processing of the nodes are much lower than its capacity limit, and means of communication and technology is in development at the time lag, make the interface bandwidth and network bandwidth become bottlenecks in the system often. Then due to the imbalance in the communication and computation, solve the communication rate and improve the communication medium than to consider how to make communication and computing power coordinated operation has more practical meaning. In fact, through the improvement of communication network and the transmission medium in recent years, Communication equipment processing capacity increase rapidly, alleviate certain communication bottleneck. But with huge amounts of data automation control system, such as a typical distribution automation system development needs, traffic and scale at the same time and communication rate data form an alternating upward trend. Such a single communication bottleneck problem, slowly into the communication and computing capabilities of the problem. Due to greatly improve the communication efficiency and the data scale, but in the data processing nodes, especially the master station and acquisition substation data processing capability of the by effects from the hardware address bus to software data concurrency, synchronization and structured problems, the quantitative qualitative change caused by that the nonlinear sharply decline. There are two aspects to consider. On the one hand, how to evaluate the known node calculation system of communication and coordination ability of "redundancy" and the possibility of potential process bottleneck. And amount of data in the current system status and requirements, configuration of communication ability and the ability to calculate coordinated way in order to achieve its work can meet the requirements, without doing some aspects of a single transformation but does not improve the overall synergy of unnecessary waste of resources. On the other hand, need according to the actual match point business data model, types, characteristics to improve the optimization of intermediate transmission communication network equipment scheduling methods and strategies, and optimize algorithm data processing nodes of massive data processing concurrent access synchronization, site search algorithm, which based on the existing hardware and resources input, do for the actual situation, play a maximum efficiency, enhance the price.

The collaborative work of communication and computation ability, needs to focus on are request response matching between computing resources, communication resources matching and computing resources through the communication between the resources and the other end of the computing resources after rate, rhythm and matching. Because each node in the system capacity and 
processing power have the limit, the running each action may need to be coordinated with other devices, so that in the process of the lack of coordination and expected to run unilaterally waiting may cause consumes a large amount of available computing and communication capabilities. A typical performance, independent terminal data acquisition speed, processing speed, calibration and other relative to receive data of master station is very slow. Without any coordination mechanism, in a cycle of a single terminal data necessary waiting time for the main needs to receive tens of millions of terminal data at the same time, the waste is very luxury. According to the statistical law, the system will not always be in a state of high load, always tend to ease after a period of time. Then according to this characteristic, the high load period failed to deal with the data cache down, guarantee the important data in advance, for the whole load is lower than the system processing capacity in the system, then send already cached data and processing. Such as In the case of data sampling deadline to allow, In the terminal equipment setting buffer cache more data after a certain period of time, and at the right time to host (or frontend). This techniques can be very effective to improve the overall system efficiency and throughput. Mismatch problems sometimes occur between communication devices and computing devices. Such as sometimes happens from time to time of emergency and non-emergency data, may be a sudden instantaneous flow rate is very high, then it can take many buffer processing technology. For emergency data volume is not big, can be in in network equipment to establish a buffer pool, to line up, so that you can avoid sending node performance degradation. For the amount of data is very big, strong timeliness and non-urgent data, can be in after sending node through the detection of network congestion, decide whether to cache on the sending terminal nodes.

\section{Fusion architecture for heterogeneous communication networks in the typical application scenarios of smart grid}

\section{1 power line carrier communication technology}

Power line carrier communication technology, hereinafter referred to as PLC, refers to the use of the existing distribution network as a transmission medium, to achieve data transmission and information exchange of a technology. The technology appeared in the early last century, the main application of power line transmission telephone sign [8]. Generalized PLC technology contains two large branches, is for the automation of distribution network, referred to as DLC (power line carrier); other one is oriented to user lines and indoor line, said PLC.

The advantage of it is that it uses the existing low voltage power network as the channel of information transmission, so that it does not need to carry on the investment and construction of the new communication network. The core issue of low voltage power line carrier communication is a carrier signal modulation and demodulation, that is, power carrier modulation and demodulation chips. With the development of low voltage power carrier communication technology, the rate of power carrier communication, the amount of data transmission and anti-interference ability have been greatly improved, which laid the material foundation for the market of power carrier communication [7-8].

Considering the above factors, Suggestions for the construction of the power distribution network is given priority to with optical fiber private network communication, broadband wireless private network and medium voltage power line carrier communication technology as a supplement. In some parts of the electric power communication private network can't cover area, can use communication operators of GPRS/CDMA / 3G public communication as a supplement, but public communication mode should be limited to not need the function of remote control terminal communications applications [8].

From the foregoing, in a typical intelligent distribution network in heterogeneous converged network there are a variety of access networks. For example: the GPRS / CDMA / 3G communication technology for wireless networks, industrial Ethernet and power line carrier communication network, the realization of the multi network fusion. In a heterogeneous network coverage range, service terminal can be according to the various access network real-time performance guarantee QoS. Through the network of the received signal strength to ensure the 
reliability of the service, and connecting with the actual business needs to select the most appropriate communication network to realize the transmission of information, avoids the single under network environment, due to the heavy network load and delay the business, such as blocking probability. With the key technology of QoS guarantee and communication capacity research is the subject of heterogeneous network integration [10]. The complexity of the heterogeneous network resources, the state of the network diversity, characteristics of the differences of the network has brought the certain challenges.

\section{2 heterogeneous fusion architecture for smart distribution networks}

Table 1 Comparison of the scheme of distribution net-work communication network

\begin{tabular}{|c|c|c|}
\hline Networking technology & advantages & disadvantages \\
\hline Optical fiber private network & $\begin{array}{l}\text { 1、 High bandwidth, higher system } \\
\text { capacity, } \\
\text { 2、High Reliability, good real-time } \\
\text { operation }\end{array}$ & 1、The construction cost is high \\
\hline $\begin{array}{l}\text { medium voltage power line } \\
\text { communication }\end{array}$ & $\begin{array}{l}\text { 1、The construction cost is low } \\
\text { 2、 Construction is simple, do not need to } \\
\text { wiring } \\
\text { 3、 Private network operation, high safety }\end{array}$ & $\begin{array}{l}\text { 1、 Low bandwidth } \\
\text { 2、 Reliability is not high }\end{array}$ \\
\hline Broadband wireless private network & $\begin{array}{l}\text { 1、 High bandwidth, large system } \\
\text { capacity, coverage, good } \\
\text { expansibility } \\
\text { 2、 Construction is simple, do not need to } \\
\text { wiring }\end{array}$ & $\begin{array}{l}\text { 1、 Frequency point problem } \\
\text { 2、 Communication effect is affected } \\
\text { by the terrain }\end{array}$ \\
\hline $\begin{array}{l}\text { Wireless narrow-band private } \\
\text { network }\end{array}$ & $\begin{array}{l}\text { 1、There are } 230 \mathrm{MHZ} \text { electric power } \\
\text { special frequency point } \\
\text { 2、The construction cost is low } \\
\text { 3、 Construction is simple, do not need to } \\
\text { wiring }\end{array}$ & $\begin{array}{l}\text { 1. Low bandwidth } \\
\text { 2、 Communication effect is affected } \\
\text { by the terrain }\end{array}$ \\
\hline wireless public network & $\begin{array}{l}\text { 1、The construction cost is low } \\
\text { 2、 Construction is simple, do not need to } \\
\text { wiring } \\
\text { 3、 Wide application and high availability }\end{array}$ & $\begin{array}{l}\text { 1、 high operating cost } \\
\text { 2、 Low real-time and security }\end{array}$ \\
\hline
\end{tabular}

Physics and a system structure of smart distribution network determines the possibility of the existence of a variety of heterogeneous network at the same time in the communication system. The optical fiber and power line carrier communication and wireless communication network is a typical application, this switch (Group), a variety of other protocol AP, gateway between network optimization became important issue in smart distribution grid is needed to study.

\section{Communication and computing collaboration key technology simulation}

Communication system's overall size, actual details of the diversity, and communication system to a variety of research and key technology if into the actual operation of the system to test and improve the work, the cost and efficiency will be difficult to expect [10]. And the application of simulation technology, the establishment of communication system simulation model, can simulate the working process of the communication system, various parameters of test system, rerun the simulation model, the reliability of the test system design, effectiveness, adaptability and stability performance [11]. Communication system simulation has become an important means in the modern communication system. Compared with other system simulation, the simulation of communication system has the following characteristics:

1) Structure and function

Communication system itself has a certain complexity, not only the types of signal source and signal processing function, the structure and scale also vary with the change of application requirement. Such as short distance wire communication and wireless communication, the difference of transmission medium may lead to different treatments on the source, the former tend to use the baseband transmission, the system is relatively simple [12]; while the latter usually need to the signal modulation before sending. After receiving the signal must go through the 
corresponding demodulation, thus system is relatively complex.

So support communication system simulation platform should have strong adaptability and flexibility. With the rapid development of modern communication technology, it is necessary to promote the optimization of the existing communication system, as well as the research and development of new communication system. Communication system simulation as an important means of research, should have a good scalability, in order to adapt to the requirements of the development of communication systems [13].

2) Large volume of data

The processing object of the communication system is a large number of signals. Communication system must be based on the transmission conditions, transmission media, transmission mode and other factors, the signal is converted into a suitable form of transmission, to ensure the accuracy and efficiency of transmission. Among them, the transmission of digital signal has high reliability, strong anti-interference ability, good security, easy to combine with the computer technology for storage and processing and so on. Therefore, even the analog signal transmission, in order to get better communication quality, and used to convert it into digital signal transmission.

But the transmission of digital signals has a great disadvantage, that is, to take up a wide communication band. For example, a channel simulate the phone only $4 \mathrm{~Hz}$ bandwidth, and a channel digital phone will have to occupy $64 \mathrm{Hkz}$ bandwidth. TV signals in general as long as the $6 \mathrm{MHz}$ bandwidth, and digital TV signals to occupy about $100 \mathrm{MHz}$ of bandwidth. In satellite communications, optical fiber communication, etc. Large capacity, broadband communication system development and increasingly mature, the shortcomings are becoming not particularly outstanding. But on communication system simulation, but can't avoid this problem.

The physical quantities (such as amplitude, time, etc.) in a communication system are often changing continuously, but only discrete data can be processed in a computer. Therefore, in the use of computer simulation, first of all make the signal in communication system to digitize. In the simulation system, these the digital data were called as the sample signals. In order to fully reflect the characteristics of the signal samples, it is often used to sample the signal with high frequency. It also can reduce the distortion introduced in the data processing, but the cost is greatly increased the amount of data needed to process.

Give a simple example: the single digital voice signal (data rate is generally 64Kbps) as the source, the modulation of the carrier frequency is cf. After digitizing, the carrier signal data rate is $4 \mathrm{cf}$. In order to maintain the spectral characteristics of the sampled signal, ef is more than 10 times of the source frequency. Therefore, the signal sample data of the signal source signal is obtained by the simulation system, at least $64 \mathrm{KbpS}^{*} 10 * 4=2560 \mathrm{Kbps}$. Each signal sample must be represented by at least one volume, and that is, the amount of data per second is at least $256^{*} 10$. A real amount is usually 64bit double precision floating point double. So the data transmission and processing of the simulation module will reach $163.84 \mathrm{Mbps}$. In the application of the control based simulation system, such as a power plant training simulator, the maximum amount of data require for the transmission of the data is designed to be about 15000 per second.

It can be seen that in the simulation process of communication system, the amount of data transmitted between the simulation modules is very large. To achieve more realistic communication system simulation, it needs to use a higher sampling rate. In this way, the amount of data transmitted is greater, the simulation modules need to deal with more data, the simulation time spent is longer.

Simulation technology in support of the theory and method research achievements inspection and the specific implementation of the construction project before the discussion aspect has the very good effect. In the operation of the simulation based on the actual need to focus on, important environmental and parameter information of simulation implementation consideration within the scope of the various actual factors to study the effect of the content of the experiment environment. Thus to support the feasibility of the theory. The typical structure of communication simulation system is shown in the following figure: 


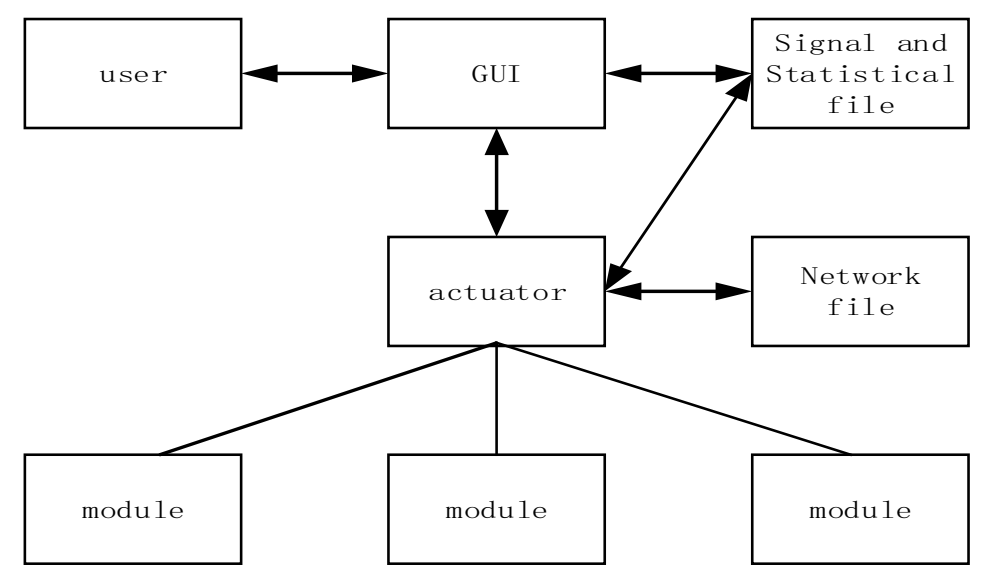

Fig. 4 Communication simulation system structure

The functions of actuator are: reading the information of the simulation example, recognition system model, establishing the internal connection between the models, reading the model parameters, arranging visit order of the models, delivering data between models, executing simulation [14].

The user access to the actuator via GUI. The actuator adopts data driven. After the beginning of the simulation, the actuator according to a certain order scheduling module in the simulation system, until the module processing the received data stream, and indicates the end of the simulation.

The communication system simulation model based system model on the complex envelope method, important sampling method, sign and system principle. The so-called "system model" is the model with the actual system corresponding to the variables, and combined with the actual system structure and parameters. System model is developed by applying the laws and principles that have been established, and the hypothesis of system structure and function. It is the explanation and reflection of the essence of the actual system and the complex cause and effect relationship. Models are high precision, various types, large in quantity. The total number is more than 280; model library has 5 categories: signal source class, communication system class, communication interference class, channel class and analysis evaluation shows [14].

System simulation software provides two performance/efficiency evaluation methods: waveform/signal diagram and statistical graph. Both of these two methods can be displayed in real time in the simulation process, and can also be stored in the file, and then processed and analyzed. Waveform/signal diagram is used to display the time and frequency characteristics of the communication signals, and the performance and efficiency of the communication can be observed in real time. Waveform/signal including time domain waveform (Baseband waveform, Band-pass waveform), eye pattern , Amplitude spectra of frequency domain, phase spectrum, power spectrum diagram, the complex domain of the in-phase component diagram, orthogonal component diagram, the signal amplitude diagram, the signal phase diagram, the constellation and so on. Statistical graph is used to display the performance/efficiency statistics of communication or jamming after each simulation. For the digital signal, the error rate and block error rate are used to represent the communication performance. For voice signal, using the intelligibility said communication performance.

So to establish an intelligent distribution network communication system simulation training environment is of great practical significance. And by using data obtained from the simulation results can be provided to the readjustment and optimization of the simulation platform to platform, at the same time, there are research results feedback improvement. The simulation platform system has two meanings, first of all, in order to provide the function of experimental platform for a variety of methods and techniques can do a simulation system based on the software. The operation characteristics of various heterogeneous network parameters and environmental impact comprehensive consideration to simulate all kinds of network running status, support technical effect test. In addition, for the intelligent power distribution system, can also build a host of highly fitting to the actual experimental environment. Through multi-threaded simulation more than 100 sets of terminals and actual conforms to the real form of heterogeneous network connection. The 
actual system and the technical effect of the simulation test are almost close to the actual situation.

\section{Summary and Prospect}

This paper mainly discusses the cooperative operation model of intelligent power distribution system communication and computing, and a collaborative simulation platform for the communication and computation of the typical application environment and the scene of intelligent distribution network. Research on the theory and key technology of the communication scheduling and computing power. The integration of smart grid and computing collaboration technology is the development trend of the future power grid system, which has incomparable advantages and broad prospects for development, and it is the inevitable direction of the future development of China's power industry. The smart grid related technologies in China is still in the exploratory stage, so the future development of space is also very broad.

\section{Acknowledgement}

In this paper, the research was sponsored by the State grid corporation of science and technology projects (Project No. SGIT0000KJJS1500010)

\section{References}

[1] R Fan Jianhui. Research on digital distribution network communication planning [D].Beijing: China Electric Power Research Institute.

[2] Kong Xiaohong. Research on task scheduling based on Intelligent Algorithms in network computing environment[D].Wuxi: Jiangnan University,2007.

[3] Yang Zhijie. How can telecommunication and electric power work hand in hand[J]. Economic \& Trade, 2010(3):21-22.

[4] Tang Hui. A review of gas distributed energy system[J]. Journal of Shenyang Institute of Engineering: Natural Science

[5] Q / GDW 378.1-2009, Power user electric energy data acquire system design guideline: Master station software[S].2009.

[6] Liu Bi. Design of feeder terminal based on DSP[D].Nanjing: Nanjing University of Aeronautics and Astronautics,2003.

[7] State Grid. General design of distribution automation communication system[R].Beijing:2013.

[8] Wang Yongfa, Yu Yongzhong, Huang Haiqing, Huang Yukui. Analysis on the current situation of low voltage power line carrier communication technology[J]. China CIO News, 2013(9):110-112.

[9] Shi Zhaoxiang, Wang Shiming. Research on simulation platform of communication systems[J]. Computer Simulation, 2000, 17(3):51-54.

[10] Yuan quan, Shi Zhaoxiang. Realization of the Communication among Simulation Objects by Using Design Pattern[J]. Computer Simulation, 2005, 22(10):15-19.

[11] Qiu Yonghong. Analysis on Activation and Decision-Making of Communication Anti-Jamming Techniques [J]. Telecommunication Engineering, 2005, 45(5):14-17.

[12] Xia Weiwei, Shen Lianfeng. QoS and Communications Capacity in Heterogeneous Networks Convergence[J]. ZTE COMMUNICATIONS, 2008, 14(3):5-8.

[13] Shi Zhaoxiang, Wang Shiming. Simulation of communication jamming effectiveness analysis[J]. Electronic Countermeasure, 2000(1):36-41.

[14] Li Yuling, Zhang Yanhua. Simulation Platform Design of Communication Systems Based on MATLAB[J]. Application Research of Computers, 2006, 23(10):248-250. 